\title{
Estados de cosas negativos La perspectiva fenomenológica de Adolf Reinach
}

\author{
MARIANO CRESPO SESMERO \\ Universidad de Navarra (España) \\ mjcrespo@unav.es
}

\begin{abstract}
Resumen
Los análisis de Adolf Reinach, fenomenólogo de la primera hora, acerca de los estados de cosas negativos se enmarcan dentro de la problemática más general de la relación entre Lógica y Ontología. Su idea de una teoría a priori del objeto, en cuanto disciplina que debe estudiar todas las clases posibles de objetos, le llevó al descubrimiento de una región de entidades revestidas de una necesidad interna y de una inteligibilidad incomparable que constituyen la esfera de lo a priori en sentido propio. Esta labor de Reinach conlleva (a) una ampliación de la esfera de lo a priori, la cual va más allá de lo meramente formal y (b) una lucha contra la subjetivización de esta esfera. La temprana muerte de Reinach en el frente durante la Primera Gran Guerra le impidió la sistematización de muchas de sus consideraciones. En este trabajo se reconstruye críticamente su teoría de los estados de cosas negativos y se plantean las líneas centrales de la crítica realizada por Roman Ingarden.
\end{abstract}

Palabras claves: Reinach, estados de cosas, fenomenología, lógica, ontología.

\section{On Negative States of Affairs \\ The Phenomenological Perspective of Adolf Reinach}

\begin{abstract}
Reinach's analysis on negative states of affairs belongs to the more general issue of the relationships between Logic and Ontology. His idea of an a priori theory of object, which task is to study all the possible kinds of objects, leads him to discover a region of entities which have an inner necessity and a incomparable intelligibility and which constitute the region of the a priori in the proper sense. This involves (a) an enlargement of the sphere of the a priori, which goes farther than the merely formal and (b) a criticism of those positions which have as consequence a subjectivization od this sphere. Reinach died during the First World $W$ ar at an early age and could not systematize some of his profound and insightful analysis. This paper offers a critical presentation of his theory of negative states of affairs taking into account Roman Ingarden's comments.
\end{abstract}

Key words: Reinach, states of affairs, phenomenology, logic, ontology.

Doctor en Filosofía por la Universidad Complutense de Madrid. Investigador del Instituto Cultura y Sociedad de la Universidad de Navarra (Pamplona, España). Entre sus publicaciones se pueden mencionar los libros El valor ético de la afectividad. Estudios de ética fenomenológica (2012) y El perdón. Una investigación filosófica (2004), así como los artículos recientes "En los orígenes de la sociología eidética" (2012) y "El amor como motivo ético en la fenomenología de Edmund Husserl” (2012). Es también editor de Menschenwürde: Metaphysik und Ethik (1998) y de la cuarta edición de la Logik de Alexander Pfänder (2000). 
Adolf Reinach, fenomenólogo de la primera hora, es uno de los autores que más sistemáticamente ha reflexionado sobre el juicio negativo y sobre los estados de cosas negativos ${ }^{1}$. Dicha reflexión - concentrada en su obra Teoría del juicio negativo (Reinach, 1989a), pero presente en algunos otros trabajos suyos- se enmarca dentro de un contexto más general: la clarificación de los conceptos de juicio y de estado de cosas (Sachverbalt) en general ${ }^{2}$. En este trabajo quisiera partir de este contexto más amplio para, seguidamente, hacer un resumen del tratamiento que este autor efectúa del juicio negativo y de los estados de cosas negativos. Por último, me referiré a una de las críticas más serias realizadas al planteamiento de este autor: la efectuada por Roman Ingarden.

\section{1. ¿Qué es un estado de cosas?}

Uno de los rasgos fundamentales de la «fenomenología ontológica» de Reinach consiste en sostener que existen relaciones esenciales necesarias entre el «lado de la conciencia» y «el lado de la objetividad» a que ésta se refiere. En este orden de cosas, un primer acercamiento ingenuo a esta cuestión podría llevar a pensar que el objeto del juicio es la objetividad que hace de sujeto. Sin embargo, ello no es así. El correlato objetivo del juicio es, más bien, el estado de cosas enunciado, el cual es análogo al objeto nombrado. Es, posteriormente, en un acto reflexivo en el que diferenciamos el estado de cosas del juzgar mismo al igual que distinguimos el objeto percibido de la percepción en cuanto acto. En el caso de los objetos y de los estados de cosas nos encontramos ante dos tipos de objetividades diferentes a los cuales apuntan actos distintos, a saber, el nombrar y el juzgar. Reinach defiende que sólo los estados de cosas pueden ser correlatos de los juicios criticando dos teorías que parecen pensar de modo diferente, a saber, la teoría del juicio de Brentano y la teoría aristotélica.

Como es sabido, Brentano y sus seguidores consideraban que toda objetividad puede ser juzgada. Esta afirmación tiene que ver

1 Una primera versión de este trabajo fue presentada en el Coloquio de Metafísica Analítica organizado por el Instituto de Filosofía de la Pontificia Universidad Católica de Valparaíso celebrado en Agosto de 2010.

2 «Exactamente igual que el estudio del juicio negativo supone una clarificación del concepto de juicio en general, así hemos de investigar ahora la esencia del correlato objetivo del juicio en general antes de poder esclarecer el correlato negativo. Aquí también desarrollaremos esta investigación solo tan lejos como sea indispensable para nuestros fines particulares» (Reinach, 1997: 33-34). 
esencialmente con lo que el propio Brentano entendía por juicio que equivalía a «reconocer» o «rechazar». Ahora bien, piensa Reinach, si eliminamos todas las significaciones extrañas de este término, podemos apreciar que estos actos no pueden referirse a entidades como colores, cosas materiales, vivencias, etc. No «afirmamos» un árbol o una mesa, sino que afirmamos el ser-frondoso de un árbol o el ser-extensa de una mesa (Reinach, 1997: 111-112; 1989b: 426). Ahora bien, si los objetos no pueden ser juzgados, ¿podrían ser las relaciones entre ellos los candidatos a ser los correlatos objetivos de los juicios? Reinach no cree necesario entrar en una investigación especial acerca de la relación para mostrar que esta teoría no resiste a un examen detenido. Sin embargo, hay juicios como, por ejemplo, «A es semejante a $\mathrm{B}$ » $\mathrm{O}$ «A está a la derecha de $\mathrm{B}$ » que parecen referirse a relaciones. Pero junto a este tipo de juicios hay otros - los más frecuentes- en cuyo lado objetivo no parece que encontremos una relación, por ejemplo, el juicio de la forma «A es B». La teoría tradicional sostendría que en un juicio de este tipo lo juzgado es una cierta relación entre A y B. Lo que Reinach cuestiona es justamente que esto sea así; que, por ejemplo, sea la relación de inherencia o de subsistencia lo que es «puesto» en el juicio «la rosa es roja».

Dentro de su esfuerzo por clarificar la naturaleza de los estados de cosas, Reinach distingue entre el ser rojo de la rosa y el rojo mismo. La distinción es evidente si atendemos a los enunciados que valen para cada una de estas objetividades. Así la rosa roja que está en el jardín, puede marchitarse; el ser rojo de la rosa ni está en el jardín ni tiene sentido decir que se marchita. Aquí no se trata de una mera diferencia de palabras, sino de dos objetividades diferentes. Como el propio Reinach afirma muy gráficamente:

El ser rojo de una rosa en tanto que tal no puede estar en el jardín exactamente del mismo modo como, por ejemplo, las fórmulas matemáticas en cuanto tales no pueden ser fragantes (Reinach, 1997: 113).

La rosa es una «cosa» y en cuanto tal nunca puede ser aseverada. En el juicio «la rosa es roja» el ser rojo de la rosa constituye su correlato objetivo. Es, pues, algo completamente distinto de ese objeto físico del mundo externo que llamamos rosa y de cualquier objeto en general, sea ideal o real. Se trata, pues, de un estado de cosas.

Ahora bien, los estados de cosas no son sólo los correlatos objetivos de los juicios, sino que también lo son de actos tales como preguntar y desear. Sin embargo, en el caso de la pregunta ocurre algo completamente distinto de lo que sucede en el juicio. Aunque ésta también se refiere a un estado de cosas, no reproduce un carácter 
existente de éste (como sucede en el juicio), sino que, por así decir, lo «pone libremente». En el caso de la duda acerca de un estado de cosas sucede algo semejante ${ }^{3}$. Asimismo, un estado de cosas puede ser el correlato de un acto de alegría. Así, por ejemplo, aquello que constituye el objeto de mi alegría cuando me encuentro con un amigo no es la persona misma del amigo, sino el estado de cosas consistente en encontrarme con él.

Precisamente, una de las características más significativas de los estados de cosas es justamente que pueden ser positivos o negativos. Esta diferencia no la podemos encontrar en el mundo de los objetos. No hay objetos positivos y/o negativos. De esta forma, junto al ser-b de A existe, coordinado con él, el estado de cosas no-ser b de A. La existencia de uno excluye la existencia del otro. Esto último no sucede en los objetos.

Asimismo los estados de cosas son truth-makers, son aquello que con su subsistencia «hacen» verdad una proposición. No se trata, sin embargo, de un «hacer» creativo, de una suerte de producción, sino, más bien, de una relación peculiar en virtud de la cual las proposiciones verdaderas suponen un fundamento ontológico que es responsable de su verdad.

\section{2. ¿Qué es un estado de cosas negativo?}

Una vez puesto de manifiesto que los estados de cosas son los correlatos objetivos de los juicios y una vez consideradas sus principales características, Reinach aborda directamente la cuestión del juicio negativo. Para ello parte de la distinción entre una serie de actos, como, por ejemplo, la representación y la mención en los que captamos objetividades «apuntando a ellas», y otros en los que «tomamos posición» frente a algo. Entre estos últimos se encuentran el tender a algo, la expectación por algo y la convicción, la cual es considerada por Reinach, junto con la aseveración, como uno de los sentidos posibles de «juicio».

3 Los actos de deseo están también dirigidos a estados de cosas. Así, siguiendo un ejemplo de Husserl: «(...) el deseo: el cuchillo debería estar sobre la mesa, correspondiente al juicio, concierne sin duda al cuchillo, pero en él no deseo el cuchillo, sino que el cuchillo esté sobre la mesa, que la situación sea ésta de hecho (daß die Sache so verhalte). Y es notorio que esta situación no debe confundirse con el juicio respectivo, ni menos con la representación del juicio; no se desea, en efecto, juicio ni representación alguna. La pregunta correspondiente se refiere igualmente al cuchillo; pero aquello por que se pregunta no es el cuchillo (lo que no tiene sentido), sino que lo preguntado es si efectivamente el cuchillo está sobre la mesa» (Husserl, 1985: 378). 
A diferencia de la primera clase de actos, este segundo tipo se caracteriza por admitir positividad o negatividad. Yo puedo estar expectante ante un acontecimiento próximo de dos formas: o bien temiendo que acaezca o bien deseando que venga al ser. En el primer caso, adopto una posición negativa y en el segundo, una positiva.

Lo mismo sucede en la convicción. Veámoslo con un ejemplo del propio Reinach:

Supongamos que alguien afirma que una flor es roja. Vamos al lugar en el que la flor se encuentra para convencernos a nosotros mismos y vemos que es amarilla. De este modo, después de habernos aproximado a la rosa con la pregunta de si es roja, ahora nos surge una convicción negativa frente a este estado de cosas, una 'increencia' (Unglaube) en el ser rojo de la rosa (Reinach, 1997: 109)4.

«Convicción negativa» significa para Reinach tanto la «increencia» dirigida a un estado de cosas positivo (el no creer en el ser amarilla de la rosa) como la convicción positiva en un estado de cosas negativo (el creer el no-ser-amarilla de la rosa). En el primer caso, se trata de un «volver la espalda» a algo y en el segundo de un «decir sí» con nuestra convicción al estado de cosas. En cualquier caso, uno y otro tipo conservan el momento de convicción. Es este momento el que las distingue de otras tomas de postura intelectuales como, por ejemplo, la expectación, la conjetura o la duda.

Por otra parte, independientemente de si la concepción clásica del juicio, según la cual el juicio positivo es un juntar o unir, es sostenible (Reinach piensa que ésta tiene un sentido diferente según se piense en la convicción o en la aseveración), los problemas de esta teoría surgirían al buscar un correlato objetivo a los juicios negativos. Al no «encontrarse» correlato alguno, se pensaba que la negación era algo puramente subjetivo, un modo de relación de la conciencia. Aun cuando en el caso de la convicción negativa de estados de cosas positivos, pueda admitirse que la negación afecta exclusivamente al «lado de la conciencia», la teoría aristotélica — según Reinach - fracasaría a la hora de explicar aquellos casos en que una convicción se dirige a algo negativo. A Reinach le parece evidente que lo negativo a lo que se refiere la convicción positiva del no ser-b de A no es una relación, sino un estado de cosas negativo. En este sentido, una primera diferencia importante entre los estados de cosas positivos y los negativos estriba en que los primeros pueden, por

\footnotetext{
4 Tómese aquí «increencia» en el sentido literal de no-creencia en un estado de cosas (disbelief en inglés), prescindiendo de las connotaciones de incredulidad religiosa que éste término tiene en castellano.
} 
así decir, ser «leídos» (abgelesen) de la realidad. Así, sobre la base de una intuición (sensible o categorial) de un objeto, surge simultáneamente la aprehensión de (y la creencia en) un estado de cosas apropiado. Por el contrario, un estado de cosas negativo nunca puede ser «leído» de la realidad.

Atendiendo, pues, a los dos tipos diferentes de negatividad que encontramos en la esfera del juicio y en la de los estados de cosas nos hallamos ante cuatro posibilidades:

(1) Convicción positiva de un estado de cosas positivo.

(2) Convicción negativa de un estado de cosas positivo.

(3) Convicción positiva de un estado de cosas negativo.

(4) Convicción negativa de un estado de cosas negativo.

Son (2), (3) y (4), pues, los que merecen el nombre de juicio negativo. Si dirigimos nuestra atención a los supuestos bajos los cuales surgen los juicios negativos de este tipo, encontramos importantes diferencias con respecto a los juicios positivos. Estos supuestos son, según Reinach, de orden psicológico y epistemológico, respectivamente. Veamos, en primer lugar, qué es lo que sucede en el caso de la convicción negativa. Como acabamos de decir, en ella nos encontramos:

(a) Un supuesto psicológico, a saber, una «toma de posición» intelectual (conjetura, creencia, duda, pregunta, etc.) referente a un estado de cosas S. Este supuesto es la actitud necesaria para que el juicio tenga lugar.

(b) Un supuesto epistemológico: una vez que hemos tomado tal posición, nos dirigimos a un estado de cosas $\mathrm{S}^{\prime}$ que está en conflicto con $\mathrm{S}$. Cuando aprehendemos $\mathrm{S}^{\prime}$ y este conflicto, $\mathrm{S}$ aparece ante nosotros de un modo completamente diferente (para el cual Reinach confiesa no tener un término adecuado). El segundo estado de cosas aprehendido, S', está presente ante nosotros en un modo que podemos caracterizar como «evidencia». Cuando captamos el conflicto en el cual el primer estado de cosas está con el segundo, el primero adquiere ante nosotros lo que Reinach denomina una evidencia negativa. Es sobre la base de esta evidencia negativa sobre la que surge en nosotros la correspondiente convicción negativa (Reinach, 1997: 123; DuBois, 1995: 50) ${ }^{5}$. Este

5 DuBois critica que Reinach considere como ocurriendo simultáneamente la aprehensión del estado de cosas contradictorio y la captación de esta contradicción. Esto sería particularmente verdad cuando hay un lapso de tiempo entre la aprehensión del estado de cosas originario y la aprehensión del estado de cosas contradictorio. Por su parte, Daubert pensaba que la captación del «conflicto» entre dos estados de cosas no es un juicio negativo sino un conocimiento del conflicto (Schuhmann, 1987: 232233). 
supuesto de orden epistemológico es el que ha de satisfacer una convicción negativa para adquirir certeza y justificación. Reinach lo denomina el fundamento del juicio negativo.

Veamos qué sucede en el caso de la convicción positiva de estados de cosas negativos. También aquí, piensa Reinach, son precisos ciertos presupuestos psicológicos y epistemológicos:

(a) Supuesto psicológico: en primer lugar, he de dirigir mi interés al estado de cosas negativo en cuanto tal (ponerlo en duda, cuestionarlo, etc.) para así poder efectuar un juicio sobre él. Por consiguiente, al igual que en el caso anterior, hemos de tomar una posición intelectual con respecto al estado de cosas.

(b) Supuesto epistemológico: al igual que en el caso (2), la convicción positiva de un estado de cosas negativo presupone la aprehensión de otro estado de cosas. La convicción de que «3 no es menor que 2 » sólo puede surgir sobre la base de la aprehensión de que «3 es mayor que 2». Sin embargo, aquí podemos notar ya una diferencia con respecto a la convicción negativa de estados de cosas positivos. Como vimos, en este caso se requería que fuera aprehendido un estado de cosas en contradicción con el estado de cosas positivo juzgado. En cambio en el caso que ahora nos ocupa el estado de cosas negativo que juzgamos (por ejemplo, el no-ser 2 mayor que 3 ) está en una conexión necesaria con el estado de cosas positivo contradictorio de tal modo que la subsistencia de uno está inmediatamente vinculada con la subsistencia del otro. Por tanto, la situación es aquí completamente diferente que en el caso anterior:

Anteriormente el estado de cosas (positivo) al cual se refería la convicción (negativa) era negativamente evidente en el sentido de que estaba en contradicción con el otro estado de cosas positivamente evidente. Ahora el estado de cosas (negativo), al cual se refiere la convicción (positiva) es positivamente evidente, puesto que está en una relación necesaria con el otro estado de cosas positivamente evidente (Reinach, 1997: 124).

Por último, también sería posible una convicción negativa de un estado de cosas negativo. Aquí el presupuesto psicológico sería —al igual que en los otros casos - una «toma de postura intelectual» ante el estado de cosas negativo en cuestión. El presupuesto epistemológico —al igual que en los otros casos- sería la aprehensión de un estado de cosas positivo que subyace a la convicción negativa. Como en el primer caso, este estado de cosas tiene que estar en conflicto con el estado de cosas 
juzgado, pero aquí el conflicto es una relación peculiar: los dos estados de cosas son contradictorios.

Resumiendo, podemos decir que

toda convicción positiva de un estado de cosas positivo o negativo presupone - epistemológicamente- la evidencia positiva de este estado de cosas; toda convicción negativa de un estado de cosas positivo o negativo presupone la evidencia negativa de ese estado de cosas; la evidencia positiva de un estado de cosas negativo, a su vez, la evidencia positiva de un estado de cosas positivo presupone, a su vez, la evidencia positiva de un estado de cosas positivo que está necesariamente unido con él. La evidencia negativa de un estado de cosas positivo o negativo presupone la evidencia de un estado de cosas positivo que está en conflicto con él y que en el caso de la evidencia negativa de un estado de cosas negativo ambos estados de cosas son entre sí contradictorios (Reinach, 1997: 125).

Después de los análisis a los que me acabo de referir, Reinach precisa que toda aseveración se funda en una convicción positiva. Evidentemente. Si tenemos una convicción negativa acerca de un estado de cosas, ésta ha de convertirse primero en una convicción positiva acerca del estado de cosas contradictorio antes de que una aseveración pueda surgir de ella.

También sabemos ya que en la convicción, al igual que en la aseveración, sólo un estado de cosas puede funcionar como su correlato objetivo. En este punto Reinach señala dos interesantes distinciones entre el modo de dársenos un estado de cosas en la convicción y la forma en que se nos da en la aseveración. En primer lugar, mientras que en la convicción el estado de cosas me es presente, está ante mí, en la aseveración es meramente mentado. En segundo lugar, y conectado con lo anterior, en la convicción el estado de cosas está ante mí, por así decir, «de un golpe». No hay una sucesión de actos de captación de los diversos elementos del estado de cosas, sino un solo acto en el que éste es captado como un todo. En la aseveración pasa algo diferente. Cuando se lleva a cabo se encuentran una serie de actos en los cuales los elementos del estado de cosas son mentados sucesivamente. Dicho con otras palabras, el estado de cosas no es mentado «de golpe», sino que se «construye» sobre una serie de actos sucesivos análogamente a como los elementos de una melodía se construyen en vivencias auditivas sucesivas. Claro está que estos actos no están uno junto al otro sin ninguna relación. 
Al igual que la unidad de los elementos unifica las diferentes vivencias en la audición total de la melodía, así la unidad de los elementos del estado de cosas unifica los actos del mentar en un mentar global de todo el estado de cosas. En este caso el mentar total está gobernado por el momento específico de la aseveración pero lo podía estar por el momento de la pregunta (Reinach, 1997: 126).

Por otra parte, al igual que en la convicción, en la aseveración hemos de distinguir entre juicios positivos y negativos, entre juicios del tipo «A es b» y de la forma «A no es b». Reinach llama la atención acerca de cómo la lógica tradicional ha considerado estos juicios como una aceptación o rechazo, como una aseveración o denegación, como un afirmar o un negar, etc. Piensa que este punto de vista ha pasado por alto el que tanto la convicción positiva como la negativa son ambas convicciones aunque de signo opuesto. Esto es lo que nos permite el incluir a ambas en la clase de los juicios. Sin embargo, ¿qué es lo que tienen en común la aseveración y la denegación que hace que ambas sean juicios? (Reinach, 1997: 127). Esta cuestión no puede ser respondida inmediatamente si no se tienen en cuenta lo siguiente.

En primer lugar, del mismo modo que en la esfera de la convicción, hemos de distinguir dos sentidos que el término «juicio negativo» tiene en el ámbito de la aseveración. Para ello Reinach acude a un ejemplo. Consideremos el juicio «el rey no era enérgico» en dos casos:

(a) en cuanto expresado por un historiador que reacciona contra la opinión de que el rey era enérgico. En este caso, el juicio se dirige contra el juicio positivo contradictorio. En este caso, la visión tradicional que presenta el juicio negativo como una denegación o rechazo parece plausible,

(b) en cuanto expresado de un modo puramente descriptivo en el curso de la narración histórica. En este caso se trata de una simple descripción. Aquí, a diferencia del primer caso, lo que tenemos es una simple «posición», un «aseverar».

Sin embargo, esta cuestión está lejos de ser evidente. Con el fin de arrojar mayor claridad sobre ella Reinach pasa a analizar el término «no» que, al menos externamente, es lo que diferencia al juicio negativo del positivo. Para ello, Reinach parte de la constatación del hecho de que no en todas las palabras podemos hacer la distinción entre mentar objetivo (gegenständliches Meinen) y objetividad mentada (gemeintes Gegenständlichen). Éste es el caso de los términos sincategoremáticos («y», «no», «pero», «también», etc.). Estas palabras son comprendidas en el curso del uso de las proposiciones sin que seamos capaces de decir que están guiadas por actos de mentar correlatos objetivos. Sin embargo, cuando utilizo estas palabras hay algo más que un mero uso de ellas, pero este «algo más» no 
es algo mentado por estos términos. Por tanto, ¿qué es lo que realmente corresponde a estas expresiones «sin objeto»?

Reinach restringe sus análisis a los términos sincategoremáticos «y y y «no». Aunque nuestro interés se centra en el análisis del término «no», puede ser útil esbozar las líneas básicas de la interpretación de la palabra «y». «Si digo: 'A y B son c' me refiero como sujetos a A y a B, pero no a un ' $y$ '. A pesar de ello, con el referirme a $A$ y a B no se agota todo lo que aquí tiene lugar. A y B no son sólo mentados, sino que, al mismo tiempo, están unidos el uno al otro. A este unir es aquello a lo que el 'y' corresponde. El 'y', por tanto, une, conecta» (Reinach, 1997: 128).

En primer lugar, resulta claro que la función principal de este término es la de conectar. Ahora bien, de esta función de «unir» $\mathrm{O}$ «conectar» que adscribimos al término «y»» hay que distinguir muy cuidadosamente aquello que se constituye ante nosotros en el acto de unión, a saber, la «totalidad»o «todo» «hecho» de A y B. Éste no es ni una totalidad espacial ni un todo temporal. De hecho, los objetos más heterogéneos pueden ser unidos mediante el «y».

Al igual que al término «y», al término «no» no le corresponde objetividad alguna. Sin embargo, existe una diferencia importante con respecto a la función de unir realizada por el término «y». Mientras que a ésta le corresponde tener al menos dos cosas que unir, la función de negar se ejerce en una objetividad sola.

Su lugar puede determinarse exactamente. Ni A ni b pueden ser negados, sino sólo el ser-b de A: en nuestro ejemplo la función de negación se dirige especialmente al 'es' y mediante él, al mismo tiempo, a todo el estado de cosas que es constituido, articulado y formado en el juicio $\mathrm{A}$ es $\mathrm{b}$ (Reinach, 1997: 130).

\footnotetext{
6 Ya Husserl, el maestro de Reinach, había reflexionado sobre estos términos tanto en la Filosofía de la Aritmética como en la sexta Investigación lógica. En esta última obra, el fundador del método fenomenológico pone de relieve cómo hay una serie de palabras «formales» que aluden a determinadas partes de las significaciones. Se trata de palabras como el, un, algunos, muchos, pocos, dos, es, no, que, y, o, etc. Dentro del ideal del cumplimiento perfectamente adecuado formulado en el capítulo III de esta misma sexta investigación, Husserl se pregunta qué clase de elementos son aquellos a los que se refieren estas palabras. En este sentido, llega a la conclusión de que se trata de elementos de la significación para los cuales no existe un correlato objetivo en la esfera de los objetos reales, «lo cual sólo quiere decir en la esfera de los objetos de una posible percepción sensible» (Husserl, 1985: 611). Esto no quiere decir que el origen de estas categorías sea la percepción interna. Lo que Husserl sostiene es la necesidad de ampliar el concepto de intuición y distinguir en ésta entre intuición sensible e intuición categorial. De este modo, «y» constituye una forma categorial sintáctica.
} 
Aquí hemos de distinguir entre la función, aquello en lo cual ésta se ejerce y lo que surge en el curso de esta actividad. En este caso la función de negación se refiere al «es»y, de este modo, surge el estado de cosas negativo. Dicho con otras palabras, esta función negativa que corresponde al «no» se dirige al elemento del estado de cosas que se expresa en el «es». Este «es» es negado y se convierte en un «no es». De este modo, el estado de cosas negativo surge por medio de la negación. Evidentemente, no nos encontramos estados de cosas negativos en el curso normal de nuestro pensamiento, sino que se «constituyen» para nosotros y quedan, por así decir, a nuestra disposición para que podamos volver a hacérnoslos presentes y aprehenderlos como aquello que se constituyó para nosotros mediante actos de negación.

Ahora bien, la expresión «constitución» de un estado de cosas negativo no ha de ser malinterpretada. No se trata, según Reinach, de una suerte de «producción» o creación de los estados de cosas. Los estados de cosas juzgados son «puestos» en actos de aseverar. En este sentido decimos que los estados de cosas positivos se «construyen» (aufbauen) en actos del mentar objetivo. Por su parte, los estados de cosas negativos requieren para su «construcción» que ciertos elementos mentados sean negados. En resumen «constitución» significa aquí: «no que los estados de cosas en sí mismos son creados mediante la función de la negación, sino que, por medio de esta función, se construyen en y para el mentar» (Reinach, 1997: 131). El mentar, pues, podríamos decir «pone de relieve» los estados de cosas negativos, los cuales subsisten con independencia de este mentar.

\section{La crítica de Ingarden}

Los análisis anteriores han puesto de manifiesto el desacuerdo de Reinach con aquellas teorías del juicio que sostienen que éste no es sino un unir o separar y que, en concreto, la esencia del juicio negativo no es sino «denegan». Reinach ve confirmado este desacuerdo en los análisis desarrollados acerca de las funciones desempeñadas por los términos sincategoremáticos. No es verdad, pues, que el negar sea lo específicamente característico del juicio negativo. Hay formaciones en las cuales sucede esto sin que por ello sean juicios. Así cuando al juicio «A no es b» replico diciendo «dudo mucho que A no sea b», en este caso hay ciertamente una negación, pero no podemos hablar seriamente de la presencia del juicio «A no es $\mathrm{B}$ », el cual es revocado en la segunda parte de la frase. Aquí falta justamente aquel momento que caracteriza a todo juicio (positivo y negativo), a saber, el momento de la aseveración. Lo 
mismo sucedería con ejemplos como «¿A no es b?», «Supongamos que A no fuera b...».

Tanto los juicios positivos como los negativos son «aseveraciones». Lo que sucede es que los primeros son aseveraciones en las cuales no existe ningún función de negación (como lo demuestra la ausencia del término «no» en su formulación lingǘstica), mientras que en los juicios negativos «el aseverar se dirige a un estado de cosas negativo que se constituye en la función de negación. Esta función de negación es la que hace que el juicio negativo sea juicio negativo. El momento de la aseveración es el responsable de que el juicio negativo sea un juicio» (Reinach, 1997: 131).

Resumiendo, podemos decir que, según Reinach, la negatividad no sólo afecta al lado de la conciencia sino que también tiene cabida en el lado objetivo. Aquello a lo que se refiere, por ejemplo, la convicción positiva del no-ser-b de A no es ni un objeto, ni una relación, sino un estado de cosas negativo. Junto al ser-b de A hay un no-ser b de A. Los estados de cosas negativos existirían (subsistirían), piensa Reinach, en el mismo sentido y exactamente con la misma objetividad que los estados de cosas positivos y estarían coordinados unos con los otros.

Es justamente esta igualdad entre el modo de ser de los estados de cosas positivos y el de los negativos el eje fundamental de la crítica de uno de los alumnos de Adolf Reinach, a saber, Roman Ingarden ${ }^{7}$. El punto de partida del fenomenólogo polaco es la cuestión de si a toda proposición enunciativa le corresponde un determinado estado de cosas que subsiste autónomamente o, viceversa, si a todo estado de cosas que subsiste autónomamente le corresponde una determinada proposición enunciativa. Ingarden reconoce que entre las proposiciones enunciativas $\mathrm{y}$ sus correlatos puramente intencionales existe, ciertamente, una relación. Por el contrario no existe siempre entre estas proposiciones y los estados de cosas que subsisten autónomamente. Ello lo mostraría, por ejemplo, la existencia de las proposiciones falsas y de los cuasi-juicios de las obras de arte literarias.

Ingarden echa de menos en Husserl, Reinach y los demás fenomenólogos, una distinción clara entre estados de cosas «puramente intencionales» y aquellos que tienen una autonomía ontológica frente a la conciencia (Ingarden, 1972: 134-135). Los primeros se caracterizan por tener su razón de ser en la proposición correspondiente, con lo cual pertenecen esencialmente a ella como su correlato. En ellos podríamos distinguir entre su contenido, su estructura intencional y su modo de ser.

La posición de Ingarden con respecto a los estados de cosas positivos se encuentra fundamentalmente en el $\int 53$ de Ingarden: 1965. 
Por lo que se refiere al carácter del ser del contenido puramente intencional es uno u otro según el tipo de proposición de la que se trate. Por su parte, los estados de cosas cuyo ser «sobrepasa», por así decir, la correlación intencional, existen en una esfera ontológica independiente de la proposición, tienen su «lugar ontológico» en el objeto mismo que aparece como su elemento-sujeto y son descubiertos por nosotros en el juicio. Asimismo, para existir han de cumplir una serie de condiciones que no obligan a los estados de cosas puramente intencionales. No solamente han de ajustarse a las leyes que se refieren a la constitución de toda objetividad, sino también a todas aquellas que dominan en la región objetiva a la que pertenece su elemento sujeto. A esto se añade que, si se trata de estados de cosas que se dan en un mundo contingente y fácticamente existente, deben cumplir las condiciones que expresan las leyes empíricas. Por otra parte, mientras que por razones ontológicoformales no es posible que un estado de cosas contenga elementos contradictorios entre sí, sí que lo es en los estados de cosas puramente intencionales (Ingarden, 1972: $\$ \$ 21$ y 22).

La crítica ingardeniana va dirigida fundamentalmente contra la equiparación del modo de ser de los estados de cosas positivos y negativos. Si éstos subsistieran del mismo modo que aquellos, llegaríamos, piensa Ingarden, a una serie de consecuencias absurdas, por ejemplo, a que el «ámbito ontológico» de todo objeto se dividiera en dos esferas: la esfera de los estados de cosas positivos que en él se fundan (en definitiva, lo que un objeto es) y la esfera de todos los posibles estados de cosas negativos con respecto a todo aquello que el objeto no es. De este modo los objetos estarían caracterizados tanto por lo que es como por lo que no es.

Ingarden (1965: 295ss) considera que esta equiparación de los modos de ser de los estados de cosas positivos y negativos es insostenible, porque, en última instancia, conduce a la tesis de que el ser es lo mismo que el no ser. Con esto no se quiere decir que los estados de cosas negativos posean algún tipo de entidad, sino que la solución habría que buscarla en un punto medio: si los estados de cosas negativos subsisten, su subsistir ha de ser muy diferente del de los positivos.

$\mathrm{El}$ «de no-hierro» (o «no-ser-hierro») de este papel, por ejemplo, no es más que un mero producto intencional que es en su ser relativo a la operación subjetiva del negar. Ciertamente está relacionado con algo positivo, pero es algo determinado en sí sólo aparentemente; tiene su fundamento en la totalidad de los estados de cosas positivos que existen en un objeto y en el acto del conocimiento del sujeto que concibe el objeto y que rechaza el momento correspondiente. El hecho de que este 
papel sea blanco da lugar a que el pensamiento esboce una infinidad de estados de cosas negativos: no es negro, no es amarillo, no es rojo, etc.

Por otra parte, como se recordará, Reinach había puesto de manifiesto que, a pesar de que los estados de cosas positivos y negativos subsisten en el mismo sentido, los segundos no podían ser «leídos» (abgelesen) simplemente de la realidad. Para aprehender un estado de cosas negativo era preciso la aprehensión previa de un estado de cosas positivo y ver que con éste está unido necesariamente un estado de cosas negativo. De este modo, el estado de cosas negativo se hace evidente positivamente, pero, según Ingarden, quedaría sin explicar la diferencia en el modo de ser de los estados de cosas negativos con respecto a los positivos.

Según Reinach, los estados de cosas negativos eran «constituidos»o surgían por medio de la función de negación de la cópula. Como decía hace un momento, «constitución» no significa aquí «producción» por esta función, sino «surgimiento» (aufbanen) en y para el mentar por medio de la negación. Con la utilización del término aufbauen Reinach pretendía eliminar todo aquello que sugiriera que lo que es aufgebant en el enunciado por la palabra «no» pierde independencia ontológica de la conciencia. Los estados de cosas (positivos y negativos) subsisten con independencia de la conciencia independientemente de si los aprehendemos, los pensamos, etc. Lo que sucedería es que para que los estados de cosas negativos sean accesibles al pensamiento ha de ser ejercida la función de negación expresada por la palabra «no». Por tanto, lo que hace esta función es descubrir a la conciencia lo que en el objeto en que juzgamos subsiste.

Las cuestiones que Ingarden plantea son ¿por qué es indispensable este descubrimiento en un estado de cosas negativo y no en uno positivo? ¿Por qué esta operación «ensambladora» es necesaria solamente en el caso de los estados de cosas negativos? Si los estados de cosas negativos existieran del mismo modo que los positivos, es incomprensible por qué no se pueden «leer» (ablesen) de la realidad de la misma manera que los positivos? ¿Cómo se llevaría a cabo este aufbauen por medio de la función de la negación?

La conclusión de Ingarden es que los estados de cosas negativos no están existencialmente en el mismo nivel que los positivos. Pero tampoco son meros entes de razón, porque que algo no posea una determinada característica es también un hecho, pero diferente de un hecho positivo. Son como «sombras» que, por así decir, se encuentran tras el objeto tan pronto como un foco luminoso se dirige a él (Ingarden, 1965: 308). Mediante los estados de cosas negativos el sujeto completa su conocimiento del objeto. En palabras del propio Ingarden, los estados de 
cosas negativos «son suscitados y determinados, por un lado, por la subsistencia de los correspondientes estados de cosas positivos y, por otro lado, por un sujeto cognoscitivo que está interesado por un determinado ámbito de determinaciones materiales (...). El sujeto completa intencionalmente al objeto mediante estados de cosas negativos en los cuales es puesta de relieve la separación de ciertas determinaciones materiales del objeto» (Ingarden, 1965: 308).

Los estados de cosas negativos tienen, pues, un doble fundamento ontológico: por un lado, la totalidad de los estados de cosas positivos fundados en un objeto y que excluyen una serie de propiedades como ajenas al «ámbito ontológico» de este objeto (no habría estados de cosas negativos, si no hubiera una esfera de propiedades imposibles de ser concretadas en uno y el mismo objeto) y, por otro lado, el acto de conocimiento del sujeto que capta el objeto y rechaza que ciertas propiedades o momentos se den en el objeto (Ingarden, 1965: 311).

Hasta aquí la posición de Ingarden acerca del carácter intencional de los estados de cosas negativos. A mi juicio, una lectura apresurada de las difíciles páginas de La polémica acerca de la existencia del mundo en las que se aborda la cuestión de la subsistencia o no subsistencia de los estados de cosas negativos podría suscitar la impresión de que éstos no son para el fenomenólogo polaco sino meros productos intencionales de actos contingentes de un sujeto cognoscente. Ciertamente, se trata de entidades intencionales, pero, como insinuábamos al final del párrafo anterior, podemos hablar de una cierta «gradación» o «diferencia» en el carácter de intencionales de estas entidades. Mientras que los estados de cosas que constituyen los correlatos de los juicios contenidos en una obra de arte no parecen tener una subsistencia independiente de los actos creativos del literato, no tenemos la impresión de que los estados de cosas negativos sean meras «creaciones» de un sujeto. El «ser-enjuto de Don Quijote» es un estado de cosas cuya subsistencia depende del juicio de Cervantes «Don Quijote es enjuto». Sin embargo, los estados de cosas negativos - como acertadamente ponía de relieve Ingarden - tienen un cierto anclaje en la realidad, «son suscitados por los correspondientes estados de cosas positivos». No son, pues, creaciones arbitrarias de un sujeto.

Por consiguiente, los estados de cosas negativos ocuparían, según Ingarden, un lugar intermedio entre la autonomía ontológica de los estados de cosas positivos y el carácter de «meros» productos intencionales de los estados de cosas puramente intencionales de, por ejemplo, los «cuasi-juicios» contenidos en una obra de arte literaria.

En cualquier caso, pienso que el problema de los estados de cosas negativos, aunque parezca moderno en su formulación, apunta a una 
«vieja» y debatida cuestión, a saber, el estatuto ontológico de las negaciones. Por lo que acabo de exponer, resulta difícil pensar que un estado de cosas negativo, una objetividad estructural que expresa la carencia o falta de una propiedad en un objeto sea una creación arbitraria del sujeto. Con ello no pretendo decir que la actividad subjetiva no desempeñe un papel importante en la formulación de estos estados de cosas pero, en mi opinión, la cuestión que aquí ha de debatirse es la del tipo de «anclaje» en la realidad que tiene un estado de cosas negativo. ¿Hasta qué punto una objetividad de este tipo tiene por fundamento in re el hecho de que una cierta determinación positiva no esté dada en un objeto?

Toda esta cuestión pone de relieve la necesidad de examinar el objeto en el que se funda el estado de cosas para determinar la subsistencia o no subsistencia de éste. Así, por ejemplo, sólo si conocemos qué es la culpa podremos afirmar que el estado de cosas que constituye el correlato objetivo del juicio «la culpa presupone libertad» subsiste o no ${ }^{8}$. Por consiguiente, el objeto se constituye en criterio para saber cuándo subsiste o no un estado de cosas. Pero eso es objeto de otro estudio.

\section{REFERENCIAS}

-DuBois, J. (1995). Judgment and Sachverbalt. An Introduction to Adolf Reinach's Phenomenological Realism. Dordrecht: Martinus Nijhoff.

-Husserl, E. (1975). Logische Untersuchungen. Erster Band: Prolegomena zur reinen Logik. Text der 1. und 2. Auflage. Husserliana XVIII. Der Haag: Martinus Nijhoff.

-Husserl, E. (1984). Logische Untersuchungen. Zweiter Band: Untersuchungen zur Phänomenologie und Theorie der Erkenntnis, Hua. XIX/1 y XIX/2. Der Haag: Martinus Nijhoff.

-Husserl, E. (1985). Investigaciones lógicas. (M. García Morente y J. Gaos, Trads.) Madrid: Revista de Occidente.

-Ingarden, R. (1965). Der Streit um die Existenz der Welt., Bd. II. Tl.1: Formalontologie: Form und Wesen. Tübingen: Max Niemeyer.

-Ingarden, R. (1972). Das literarische Kunstwerk. Eine Untersuchung aus dem Grenzgebiet der Ontologie, Logik und Literaturwissenschaft. 4. Aufl. Tübingen: Max Niemeyer.

-Mulligan, K. (1987). Speech Act and Sachverbalt. Reinach and the Foundations of Realist Phenomenology. Dordrecht: Martinus Nijhoff.

\footnotetext{
8 Además, en este caso, el conocimiento de ese «qué» que es la culpa hace posible también que reconozcamos el carácter necesario del estado de cosas. Como veremos en los capítulos siguientes, la explicación reside en el tipo de ser de este «qué».
} 
-Reinach, A. (1989). Sämtliche Werke. Textkritische Ausgabe in 2 Bänden. (K. Schuhman und B. Smith, Hrsg.). München, Hamden, Wien: Philosophia Verlag.

-Reinach, A. (1989a). Zur Theorie des negativen Urteils. En Münchener Philosophische Abhandlungen. Theodor Lipps zu seinem zechiigsten Geburtstag gewidmet von seinen früheren Schülern. Leipzig: Johann Ambrosius Barth, 1911 (reimpreso en Reinach 1989, págs. 95-140).

-Reinach, A. (1989b). Einleitung in die Philosophie [1913] (reimpreso en Reinach 1989, págs. 369-513).

-Reinach, A. (1997). Teoría del juicio negativo. (M. Crespo, Trad.). Madrid: Universidad Complutense.

-Schuhmann, K. (1987). Johannes Dauberts Kritik der 'Theorie des negativen Urteils' von Adolf Reinach. En K. Mulligan, K., Speech Act and Sachverhalt. Reinach and the Foundations of Realist Phenomenology (págs. 227-238). Dordrecht: Martinus Nijhoff.

Sumario: 1. ¿Qué es un estado de cosas?; 2. ¿Qué es un estado de cosas negativo?; 3. La crítica de Ingarden; Referencias. 